\title{
A tetraoxane-based antimalarial drug candidate that overcomes PfK13-C580Y dependent artemisinin resistance
}

Paul M. O’Neill1,2, Richard K. Amewu ${ }^{1, \dagger}$, Susan A. Charman ${ }^{3}$, Sunil Sabbani ${ }^{1}$, Nina F. Gnädig ${ }^{4}$, Judith Straimer ${ }^{4}$, David A. Fidock ${ }^{4,5}$, Emma R. Shore ${ }^{1}$, Natalie L. Roberts' ${ }^{1}$, Michael H.-L. Wong ${ }^{1}$, W. David Hong ${ }^{1}$, Chandrakala Pidathala', Chris Riley¹, Ben Murphy ${ }^{1}$, Ghaith Aljayyoussi ${ }^{6}$, Francisco Javier Gamo7 ${ }^{7}$ Laura Sanz ${ }^{7}$, Janneth Rodrigues7, Carolina Gonzalez Cortes ${ }^{7}$, Esperanza Herreros ${ }^{7}$, Iñigo Angulo-Barturén7, María Belén Jiménez-Díaz ${ }^{7}$, Santiago Ferrer Bazaga7, María Santos Martínez-Martínez ${ }^{7}$, Brice Campo ${ }^{8}$, Raman Sharma ${ }^{6}$, Eileen Ryan ${ }^{3}$, David M. Shackleford ${ }^{3}$, Simon Campbell ${ }^{8}$, Dennis A. Smith ${ }^{8}$, Grennady Wirjanata9 Rintis Noviyanti ${ }^{10}$, Ric N. Price ${ }^{9,11}$, Jutta Marfurt ${ }^{9}$, Michael J. Palmer ${ }^{8}$, lan M. Copple ${ }^{2}$, Amy E. Mercer ${ }^{2}$, Andrea Ruecker ${ }^{12}$, Michael J. Delves ${ }^{12}$, Robert E. Sinden ${ }^{12,13}$, Peter Siegl ${ }^{8}$, Jill Davies ${ }^{6, \dagger}$, Rosemary Rochford ${ }^{14}$, Clemens H.M. Kocken ${ }^{15}$, Anne-Marie Zeeman ${ }^{15}$, Gemma L. Nixon', Giancarlo A. Biagini ${ }^{6}$ \& Stephen A. Ward ${ }^{6}$

K13 gene mutations are a primary marker of artemisinin resistance in Plasmodium falciparum malaria that threatens the long-term clinical utility of artemisinin-based combination therapies, the cornerstone of modern day malaria treatment. Here we describe a multinational drug discovery programme that has delivered a synthetic tetraoxane-based molecule, E209, which meets key requirements of the Medicines for Malaria Venture drug candidate profiles. E209 has potent nanomolar inhibitory activity against multiple strains of $P$. falciparum and $P$. vivax in vitro, is efficacious against $P$. falciparum in in vivo rodent models, produces parasite reduction ratios equivalent to dihydroartemisinin and has pharmacokinetic and pharmacodynamic characteristics compatible with a single-dose cure. In vitro studies with transgenic parasites expressing variant forms of $\mathrm{K} 13$ show no cross-resistance with the C580Y mutation, the primary variant observed in Southeast Asia. E209 is a superior next generation endoperoxide with combined pharmacokinetic and pharmacodynamic features that overcome the liabilities of artemisinin derivatives.

\footnotetext{
${ }^{1}$ Department of Chemistry, University of Liverpool, Liverpool L69 7ZD, UK. ${ }^{2}$ Department of Pharmacology, School of Biomedical Sciences, MRC Centre for Drug Safety Science, University of Liverpool, Liverpool L69 3GE UK. ${ }^{3}$ Centre for Drug Candidate Optimisation, Monash Institute of Pharmaceutical Sciences, Monash University, 381 Royal Parade, Parkville, Victoria 3052, Australia. ${ }^{4}$ Department of Microbiology and Immunology, Columbia University College of Physicians and Surgeons, HHSC 1502, 701 W. 169th Street, New York, New York 10032, USA. 5 Division of Infectious Diseases, Department of Medicine, Columbia University Medical Center, HHSC 1502, 701 W. 168th Street, New York, New York 10032, USA. 6 Research Centre for Drugs and Diagnostics, Liverpool School of Tropical Medicine, Pembroke Place, Liverpool L3 5QA, UK. ${ }^{7}$ Tres Cantos Medicines Development Campus, DDW, GlaxoSmithKline, Severo Ochoa 2, 28760 Tres Cantos, Spain. ${ }^{8}$ Medicines for Malaria Venture, ICC, Route de Pré-Bois 20, P.O. Box 1826, 1215 Geneva, Switzerland.

${ }^{9}$ Global and Tropical Health Division, Menzies School of Health Research, Charles Darwin University, P.O. Box 41096, Casuarina, Darwin, Northern Territory 0811, Australia. ${ }^{10}$ Eijkman Institute for Molecular Biology, Jl. Diponegoro 69, 10430 Jakarta, Indonesia. ${ }^{11}$ Nuffield Department of Clinical Medicine, Centre for Tropical Medicine and Global Health, University of Oxford, Old Road Campus, Roosevelt Drive, Oxford OX3 7FZ, UK. ${ }^{12}$ Department of Life Sciences, Imperial College London, South Kensington, London SW7 2AZ, UK. ${ }^{13}$ Nuffield Department of Medicine, The Jenner Institute, University of Oxford, Old Road Campus Research Building, Roosevelt Drive, Oxford, OX3 7DQ, UK. ${ }^{14}$ Department of Immunology and Microbiology, University of Colorado, Aurora Colorado, CO 80045, USA. ${ }^{15}$ Department of Parasitology, Biomedical Primate Research Centre, P. O. Box 3306, 2280 GH Rijswijk, The Netherlands. $\dagger$ Present address: Department of Chemistry, University of Ghana, P.O. Box LG56, Legon, Accra, Ghana. Correspondence and requests for materials should be addressed to P.M.O'N. (email: pmoneill@liv.ac.uk).
} 
T he control and elimination of malaria requires effective treatment strategies and the universal availability of artemisinin-based combination therapies $(\mathrm{ACTs})^{1,2}$. For over a decade, research groups have sought to replace the semisynthetic artemisinin components (Fig. 1a, 1a-1c) of ACTs with a fully synthetic alternative to reduce uncertainties regarding cost and supply and to improve overall pharmacological qualities, most notably short clinical half-lives. Several pharmacophores have been explored ${ }^{3}$ including the tetraoxanes (Fig. 1b, 3a and $\mathbf{3 b}$ ).

Recent years, however, have witnessed the emergence of parasite 'resistance' to artemisinin, first documented in western Cambodia ${ }^{4,5}$. Malaria patients from this region were reported to have a delayed parasite clearance following either artesunate monotherapy or an ACT. The delayed parasite clearance phenotype is not resistance as defined by the $\mathrm{WHO}^{6,7}$ and does not necessarily lead to treatment failure ${ }^{8}$. However, it gives rise to concerns of a reduction in the therapeutic lifespan of the most effective, currently registered anti-malarials and the only class that offers rapid parasite biomass reduction. Furthermore, reduced artemisinin efficacy places greater selective pressure on the ACT partner drugs, increasing the risk of multidrug resistance to emerge. Indeed, recent results in Cambodia now document increasing rates of clinical treatment failure in patients treated with the first-line combination dihydroartemisinin plus piperaquine, because of the emergence of resistance to both agents 9

Here we describe the development of a fully synthetic tetraoxane analogue, E209, which displays nanomolar efficacy against multiple strains of $P$. falciparum and $P$. vivax with a tailored combination of pharmacodynamic (PD) and pharmacokinetic $(\mathrm{PK})$ properties compatible with a single-dose cure even against a backdrop of the K13-dependent resistance mechanism in operation in S.E. Asia ${ }^{8}$.

\section{Results}

Optimization of the tetraoxane series. The tetraoxane core $3 \mathbf{a}$ is differentiated from 1,2,4-trioxolanes $\mathbf{2 a}$ and 1,2,4-trioxanes as it is achiral, has greater inherent thermodynamic stability and potent in vitro antimalarial activity, either as a simple unsubstituted analogue, for example, 3a (Fig. 1) or related derivatives. Recent theoretical calculations based on stereoelectronic analysis suggest that the remarkable thermodynamic stability of tetraoxanes can be attributed to a stereoelectronic 'double anomeric effect' which stabilizes the six-membered ring system ${ }^{10}$.

Our initial medicinal chemistry focus was the preparation of variants of a simplified analogue of our previous candidate RKA 182 with more balanced ADME properties $(\mathbf{3 b})^{11}$ (Supplementary Figs 1, 2, 3 and 4). Multiple series were prepared to modulate solubility, metabolic stability, pKa and log D (Supplementary Fig. 4) but in all cases, the amide linked analogues, although superior to the artemisinins, had relatively short half-lives and poor antimalarial activity in the Plasmodium berghei mouse model. We next prepared tetraoxane $\mathbf{4}$ as a direct comparator (Supplementary Fig. 4; Supplementary Table 1) to OZ439 (ref. 12), which is currently in phase 2 clinical trials. Although this analogue had equivalent in vitro potency to OZ439, it only achieved a 16.3 day mean survival time (MSD) at a single $1 \times 30 \mathrm{mg} \mathrm{kg}^{-1}$ oral dose in $P$. berghei infected mice (Table 1 ). This molecule was also slightly less stable in microsomal studies in vitro with high clearance rates and a short half-life in rat PK studies.

Cognisant of the liabilities of the artemisinin class, single-dose survival time, intrinsic clearance and blood stability were used as the key parameters to drive SAR studies towards lead series progression (Supplementary Fig. 4; Supplementary Information). Representative lead compounds are shown in Supplementary Fig. 4 from which E209 and N205 were the clear front-runner molecules with respect to cures in the $P$. berghei model and initial DMPK assessments (Supplementary Table 1; Supplementary Fig. 5) and were selected for further profiling. In vitro metabolic stability assessments (Supplementary Table 2) and solubility assessments (Supplementary Table 3) demonstrated the superior properties of E209 and this compound was taken forward.

Following scale up synthesis (Supplementary Note 2; Supplementary Fig. 1), E209 was progressed to candidate selection through a series of chemical, parasitological, pharmacological and toxicological checkpoints as outlined below.

PD properties of E209. E209 retained potent in vitro efficacy (mean $\mathrm{IC}_{50}$ range $2.9-14.0 \mathrm{nM}$ ) against a panel of 10 sensitive and multidrug-resistant $P$. falciparum parasite isolates from distinct geographical origins, with no observable cross-resistance in growth inhibition studies (Supplementary Table 4). Supplementary Table 4 represents a panel of parasite isolates used as part of a standard Medicines for Malaria Venture (MMV) in vitro evaluation and includes parasites that are universally sensitive to current drugs (HB3) and parasites resistant to chloroquine (as indicated), mefloquine (FCB, NF54), pyrimethamine and related DHFR inhibitors (K1, TMC90, V1/S and so on) and atovaquone (TMC90). In the case of E209, activity against all parasites is below the accepted cut off for resistance and it is clear from visual inspection that there is no relationship at all with chloroquine sensitivity. Cytotoxicity studies using HeLa cells demonstrated that E209 had a high in vitro therapeutic index of $200-1,000$, with a median $\mathrm{IC}_{50} 2.85$ (s.d. $\pm 0.99 \mu \mathrm{M}$, $n=3$ ) (c.f. dihydroartemisinin cytotoxicity (median $\mathrm{IC}_{50} 0.4$ (s.d. $\pm 0.1 \mu \mathrm{M}, n=3)$ ).

Ex vivo $P$. falciparum and $P$. vivax drug sensitivity experiments were carried out in Papua Province in eastern Indonesia, an area with documented multidrug-resistant $P$. falciparum and CQresistant $P$. vivax, using a modified schizont maturation assay as described previously ${ }^{13-17}$. Ethical approval for the ex vivo $P$. falciparum and $P$. vivax efficacy data was obtained from the Human Research Ethics Committee of the NT Department of Health \& Families and Menzies School of Health Research, Darwin, Australia (HREC 2010-1396) and the Eijkman Institute Research Ethics Commission, Jakarta, Indonesia (EIREC 47 and EIREC 67). E209 exhibited equipotent ex vivo activity against $P$. vivax $\left(n=13\right.$; median $\left.\mathrm{IC}_{50}: 10.5 \mathrm{nM}\right)$ and $P$. falciparum $(n=3$; median $\left.\mathrm{IC}_{50}: 15.7 \mathrm{nM}\right)$ clinical field isolates from Papua, Indonesia (Mann-Whitney $U$ test, $P=0.570$ ); Supplementary Table 5. With the exception of chloroquine in $P$. falciparum (Spearman rank correlation, $P=0.042$ ), there was no correlation between the activity of E209 and any of the other anti-malarials assessed in either species ((amodiaquine (AQ), piperaquine (PIP), mefloquine (MFQ) and artesunate (AS) (Supplementary Table 6)).

E209 was screened for late stage gametocytocidal activity in a standard membrane feeding assay (SMFA) and transmissionblocking activity was determined in Anopheles stephensi mosquitoes. Late stage gametocytes were incubated with E209 for $24 \mathrm{~h}$ prior to mosquito feeding and the $\mathrm{IC}_{50}$, (determined by the reduction in oocyst mean intensity) was $14.5 \mathrm{nM}$ with a corresponding $\sim 60$ and $90 \%$ transmission-blocking activity (defined as the percentage of mosquitoes with no observed oocysts) recorded at $100 \mathrm{nM}$ and $1 \mu \mathrm{M}$, respectively (Supplementary Fig. 6). The transmission reducing activity (TRA) shown by E209 is consistent with that shown for the endoperoxide class as reported in recent SMFA-based studies ${ }^{18,19}$. 
a

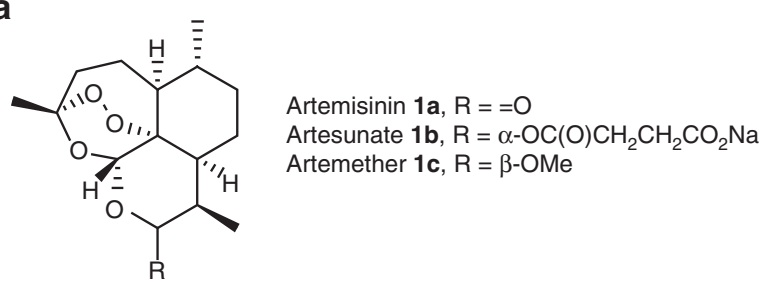

b

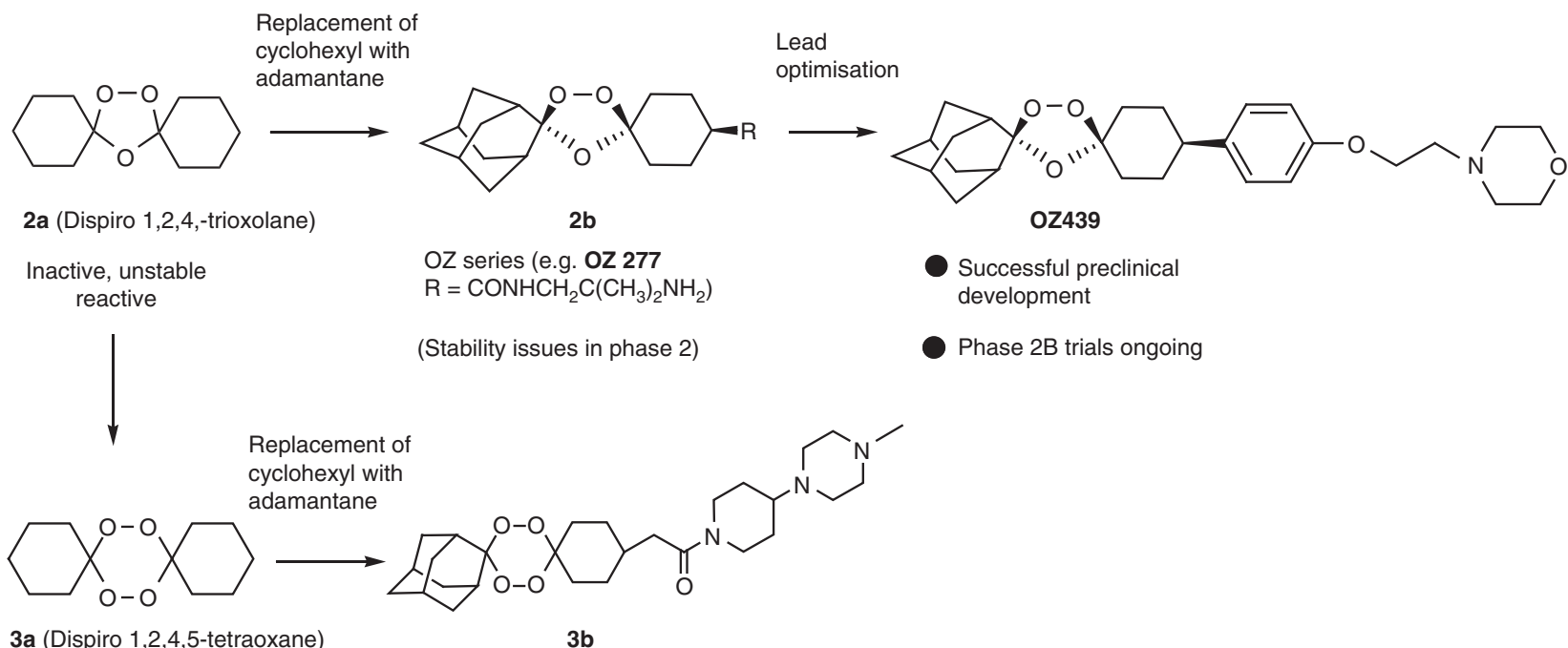

Highly potent $\mathrm{IC}_{50}=25 \mathrm{nM}$ Achiral, more stable than 1,2,4-Trioxolane $\mathbf{2 a}$
- RKA182 - Extended half-life, stability, maintained potency

- Enhanced Fe(II) stability

PK profile not compatible with single dose cure approach

Figure 1 | Artemisinins and synthetic peroxides. (a) Artemisinin and semi-synthetic analogues. (b) Comparison of tetraoxanes with trioxolane-based anti-malarials. MSD in days following a single oral dose of $30 \mathrm{mg} \mathrm{kg}^{-1}$, Pf SCID (NOD-SCID IL-2R $\gamma \mathrm{C}^{\text {null }}$ (NSG) mice engrafted with human erythrocytes and infected with $P$. falciparum strain 3D70087/N9).

E209 $\left(10 \times \mathrm{IC}_{50}\right)$ displayed fast killing kinetics (Supplementary Methods), with an in vitro parasite reduction ratio ( $\left.\log _{10} \mathrm{PRR}\right)$ $>4.8$ similar to artemisinin $\left(\log _{10} \mathrm{PRR}>4.8\right)$, and faster than CQ $\left(\log _{10}\right.$ PRR 4.5), pyrimethamine $\left(\log _{10}\right.$ PRR 3.7$)$ and atovaquone $\left(\log _{10}\right.$ PRR 2.9) (Fig. 2) 20 .

Critical for the development of E209 and related structures is the evaluation of their activity against ART-resistant parasites, which has been defined as delayed parasite clearance in patients ${ }^{5}$. This phenotype has been associated clinically with a number of nonsynonymous SNPs in the propeller domain of the K13 protein, located on chromosome 13 (ref. 23). Notably, these mutations could not be associated with ART resistance using the standard in vitro $72 \mathrm{~h}$ inhibitory assay, therefore a novel phenotypic assay was developed to assess ART resistance in vitro. The ring-stage survival assay $\left(\mathrm{RSA}_{0-3 \mathrm{~h}}\right)$ is based on the increased resistance of young ringstage parasites to a $6 \mathrm{~h}$ pulse of $700 \mathrm{nM}$ dihydroartemisinin (DHA) ${ }^{21,22}$. To investigate the impact of K13 mutations on E209 susceptibility, a series of gene-edited, otherwise isogenic parasite lines with either K13 mutant or wild-type alleles have been subjected to $\mathrm{RSA}_{0-3 \mathrm{~h}}$ assays using DHA as comparison ${ }^{23}$. This allowed us to assess the potency of a pharmacological relevant dose of E209 against K13 mutant parasites and to identify potential cross-resistance phenotypes. In this study we focused on the most prevalent mutation C580Y and R539T, which was demonstrated to confer high levels of DHA resistance in $v_{\text {itro }}{ }^{23}$ (Fig. 3).Generally, we observe higher survival rates after exposure of young ring stages to E209 in both parasite backgrounds when compared to DHA. When subjected to a $700 \mathrm{nM}$ dose for $6 \mathrm{~h}$, we observe $4 \%$ survival of V1/S and $16 \%$ of Cam3.II parasites for E209 compared to $<1 \%$ survival when pulsed with DHA. The highly prevalent K13 mutation C580Y does not confer cross-resistance to E209. However, R539T shows a moderate but significant increase of parasite survival in both parasites lines tested. (Ring-stage survival assays, fold changes between K13 wildtype and K13-mutant lines and $\mathrm{IC}_{50}$ data for DHA and $\mathrm{E} 209$ are recorded in Supplementary Tables 15 and 16; Supplementary Fig. 7).

In vivo experiments using $P$. berghei infected mice demonstrated that E209 when given orally resulted in complete parasite clearance in the Peters' standard 4-day suppressive test ${ }^{24}$, with an estimated $\mathrm{ED}_{50}$ of $4 \mathrm{mg} \mathrm{kg}^{-1}$ after three doses. Importantly, E209 also achieves $66 \%$ cure rate after a single oral dose of $30 \mathrm{mg} \mathrm{kg}^{-1}$ (Table 1). Efficacy of E209 against the human malaria parasite was tested in the immune-deficient NOD-SCID IL-2R_null mouse engrafted with human erythrocytes and infected with the P. falciparum strain $3 \mathrm{D} 7^{0087 / N 9}$ (ref. 25). Complete parasite clearance was achievable with a single oral dose of E209 $\left(30 \mathrm{mg} \mathrm{kg}^{-1}\right)$ with a calculated $\mathrm{ED}_{90}$ of $11.6 \mathrm{mg} \mathrm{kg}^{-1}$ and an AUC $\mathrm{ED}_{90}$ of $1.2 \mu \mathrm{g} \mathrm{h} \mathrm{m}^{-1}$ (Fig. 4). This compares with an $\mathrm{ED}_{90}$ of $10 \mathrm{mg} \mathrm{kg}^{-1}$ for artesunate following four consecutive daily doses $^{26}$. The maximum rate of parasite killing by E209 was achieved with blood exposure between 5 and $10 \mu \mathrm{gh} \mathrm{ml}^{-1}$ 
Table 1 | Calculated physicochemical` properties and in vitro and in vivo antimalarial activity $\ddagger$ profiles of selected tetraoxane derivatives.

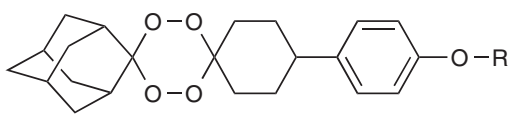

\begin{tabular}{|c|c|c|c|c|c|}
\hline $\begin{array}{l}\text { Compound } \\
\text { Number }\end{array}$ & Side Chain (R) & $C \log P$ & PSA/ $\AA^{2}$ & $\begin{array}{c}\mathrm{IC}_{50} \\
\text { (3D7) } \\
\mathrm{nM}\end{array}$ & $\begin{array}{c}\text { In vivo }(\mathrm{Pb}) \\
\text { MSD (days) at } 1 \\
\times 30 \mathrm{mg} / \mathrm{kg}\end{array}$ \\
\hline $4^{\dagger}$ & & 5.6 & 58.6 & $10 \pm 1$ & $16.3(14,21,21)$ \\
\hline $5^{\dagger}$ & & 5.9 & 58.6 & $7.5 \pm 0.2$ & $13(13,13,13)$ \\
\hline $6^{\dagger}$ & & 5.6 & 86.7 & $3.5 \pm 0.2$ & $19.3(14,30,14)$ \\
\hline $7^{\dagger}$ & & 5.9 & 86.7 & $3.7 \pm 0.1$ & $14(14,14,14)$ \\
\hline $8^{\dagger}$ & & 6.1 & 49.3 & $8.5 \pm 0.6$ & $(8,8,8(8)$ \\
\hline $\mathrm{E}^{209^{\dagger}}$ & & 6.4 & 49.3 & $5.1 \pm 0.81$ & $\begin{array}{c}25.0(15,30,30) \\
\text { ( } 2 \text { cures })\end{array}$ \\
\hline $0 Z 439^{12}$ & See Figure $1 b$ & 5.5 & 49.3 & $5.1 \pm 0.81$ & $\begin{array}{c}30(30,30,30) \\
(3 \text { cures })\end{array}$ \\
\hline
\end{tabular}

${ }^{\star} \mathrm{C} \log \mathrm{P}$ (calculated partition coefficient) and polar surface area (PSA) calculated using Chem Draw version 15.

$\dagger$ †osed in citric acid formulation. Data recorded for in vivo studies of OZ439 in the Plasmodium berghei model is taken from Charman et al. ${ }^{12}$

$\ddagger$ in vivo experiments were performed in $P$. berghei infected mice $(n=3)$. MSD refers to mean survival days following a single oral dose of $30 \mathrm{mg} \mathrm{kg}^{-1}$

(Fig. 4). Therefore, this range of exposure in human blood is predicted to be the lowest necessary to achieve the maximum parasite killing and can therefore be defined as the minimum parasiticidal concentration (MPC).

In vitro and in vivo PKs of E209. E209 exhibited metabolic degradation in human, rat and mouse liver microsomes with rates generally being fastest in rat and slowest in the mouse (Supplementary Table 17; Table 2). Comparing metabolite profiles across all three species, hydroxylation(s) on the adamantane ring to form multiple $\mathrm{M}+16$ metabolites represent important pathways in all three species. In addition, $N$-oxidation to $M+16$ appears to be an important metabolic pathway in rat whilst tetraoxane cleavage products were observed in all three species (Supplementary Tables 17, 18, 19; Supplementary Fig. 8).

Compartmental PK modelling of preclinical in vivo data showed a clear two compartmental trend for all three species. Simulated PK profiles based on these compartmental model predictions (Table 2) showed good agreement with observed data as shown in Fig. 5a-c). Allometric scaling (see Methods) rat PK data predicted a terminal half-life in humans of $24-30 \mathrm{~h}$ and drug levels that exceed E209 $\mathrm{IC}_{50}$ levels for up to 6 days following a single oral dose of $15 \mathrm{mg} \mathrm{kg}^{-1}$ (Fig. 5d).

Preclinical safety studies. To assess potential for adverse activities, we profiled E209 using a diverse panel of radioligand binding assays (CEREP screen), ion channel voltage clamp assays and genotoxicity assays. In the CEREP screen, E209 at $10 \mu \mathrm{M}$ demonstrated a favourable profile apart from moderate potency at Sigma 1 and Sigma 2 receptors ( $\mathrm{IC}_{50} \mathrm{~s} c a .200 \mathrm{nM}$ ). Risk of adverse cardiac activity including QT interval prolongation is predicted to be low based on the measurement of a high $\mathrm{IC}_{50}$ $(>3.5 \mu \mathrm{M})$ in the hERG assay and no inhibitory activity at $>100 \mu \mathrm{M}$ in Cav1.2 and Nav1.5 assays. E209 was negative in the Ames test and mouse micronucleus test confirming the absence of mutagenic potential.

We also examined the potential haemolytic effect of E209 in NOD-SCID mice engrafted with the African variant G6PD deficient human red blood cells (huRBC) as previously reported for primaquine ${ }^{27}$. Mice treated with E209 at doses up to $50 \mathrm{mg} \mathrm{kg}^{-1} \mathrm{day}^{-1}$ for 3 days did not show a significant drugdependent loss of huRBC at 7 days post-treatment with all test groups demonstrating a response similar to the vehicle control (Supplementary Fig. 9A-C). In contrast primaquine, a drug that causes haemolytic toxicity in humans with G6PD deficiency, induced significant loss of huRBC (Supplementary Fig. 9A-C).

In addition, In vitro studies with cytochrome P450 (CYP) isoforms demonstrated that E209 did not inhibit any of the major human CYP450s with $\mathrm{IC}_{50} \mathrm{~s}>20 \mu \mathrm{M}$ (Supplementary Table 20).

The maximum-tolerated dose (MTD) following a single oral administration of E209 in Sprague-Dawley rats was $300 \mathrm{mg} \mathrm{kg}^{-1}$. A 7-day repeat dose, exploratory toxicity study in rats was performed using dose levels of 50,100 and $200 \mathrm{mg} \mathrm{kg}^{-1}$ and parallel groups for toxicokinetic analysis. At $200 \mathrm{mg} \mathrm{kg}^{-1} \mathrm{day}^{-1}$ E209 was not tolerated in either sex. At $100 \mathrm{mg} \mathrm{kg}^{-1}$ there was body weight loss in females. On the basis of weight loss and histopathology findings, the no observed adverse effect level (NOAEL) for this study was $100 \mathrm{mg} \mathrm{kg}^{-1}$ day $^{-1}$ for males and $50 \mathrm{mg} \mathrm{kg}^{-1} \mathrm{day}^{-1}$ for females. Systemic 


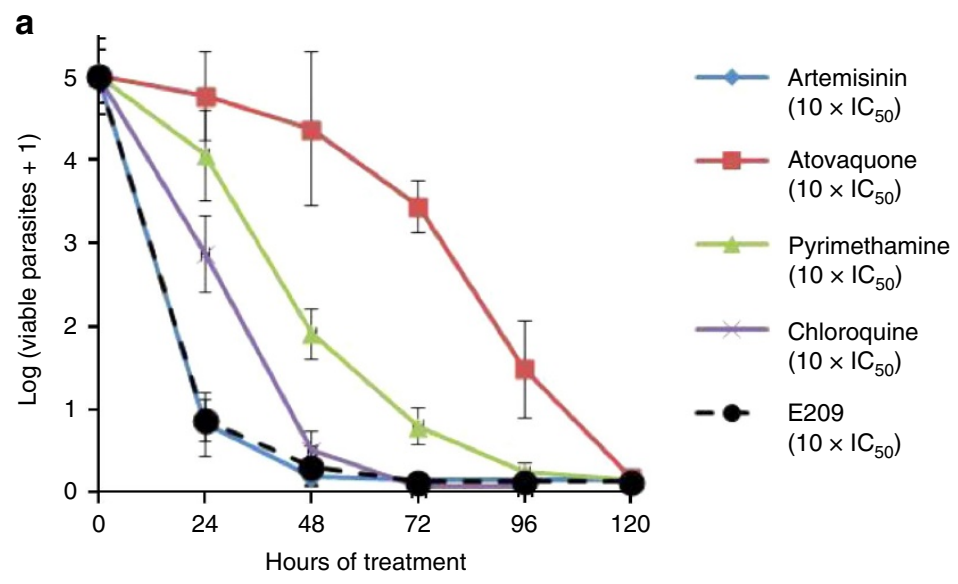

b

\begin{tabular}{|c|c|c|c|c|c|c|}
\hline Compound & Dose & $\begin{array}{c}\text { Lag phase } \\
(\mathrm{h})\end{array}$ & Slope & $R$ & Log PRR & $\begin{array}{c}\text { PCT 99.9 } \\
\%(\mathrm{~h})\end{array}$ \\
\hline Artemisinin & $10 \times \mathrm{IC}_{50}$ & 0 & -0.101 & -0.92 & $>4.8$ & $<24$ \\
\hline Atovaquone & $10 \times \mathrm{IC}_{50}$ & 48 & -0.060 & -0.992 & 2.9 & 81 \\
\hline Pyrimethamine & $10 \times \mathrm{IC}_{50}$ & 24 & -0.077 & -0.994 & 3.7 & 57 \\
\hline Chloroquine & $10 \times \mathrm{IC}_{50}$ & 0 & -0.094 & -0.999 & 4.5 & 34 \\
\hline E209 & $10 \times \mathrm{IC}_{50}$ & 0 & -0.098 & -0.915 & $>4.8$ & $<24$ \\
\hline
\end{tabular}

Figure 2 | Parasite reduction ratios of clinically used anti-malarials and E209. (a) In vitro PRR-number of viable parasites after E209 treatment $\left(10 \times \mathrm{IC}_{50}\right)$ is compared with the profile shown by standard anti-malarials (b) Lag phase, $\log _{10}$ PRR and parasite clearance time (PCT) values for E209 and a selection of standard anti-malarials. The in vitro parasite reduction rate assay was used to determine onset of action and rate of killing as previously described ${ }^{20}$. P. falciparum was exposed to E209 at a concentration corresponding to $10 \times E_{50}$. The number of viable parasites at each time point was determined as described by Sanz et al. ${ }^{20}$ Four independent serial dilutions were done with each sample to correct for experimental variation; error bars, s.d. Previous results reported on standard anti-malarials tested at $10 \times \mathrm{EC}_{50}$ using the same conditions are shown for comparison.
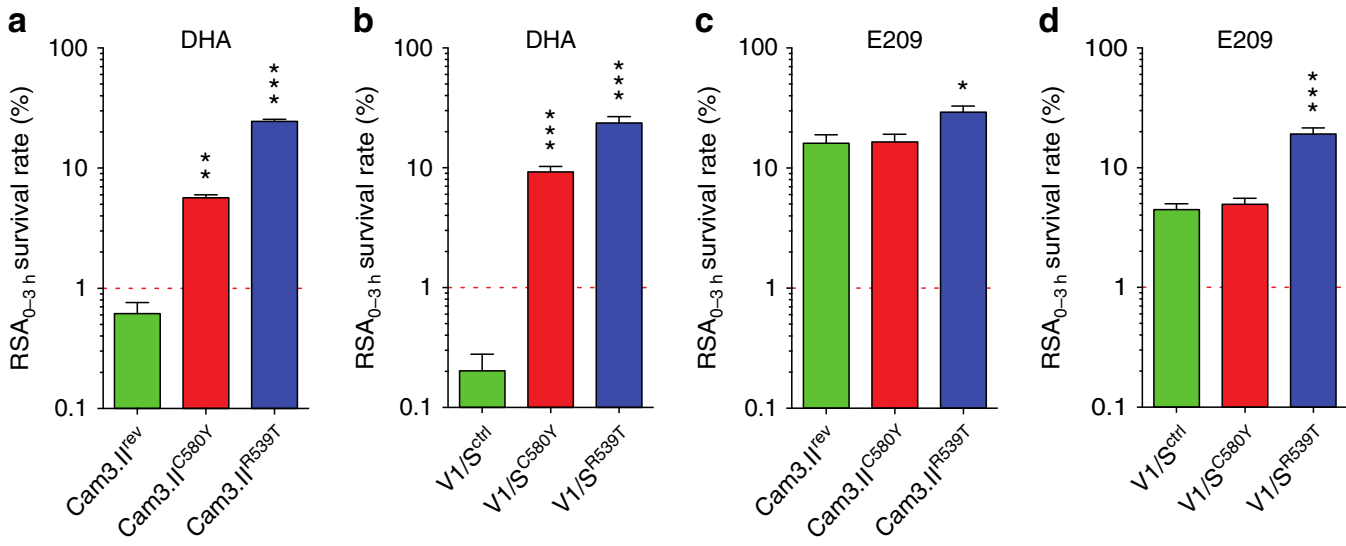

Figure 3 | Ring-stage survival assays show equal potency of E209 against K13 wild-type and C580Y mutant parasites whereas R539T confers a marginal increase of survival. Results show the percentage of early ring-stage parasites (0-3 h post-invasion of human erythrocytes) that survived a 6-h pulse of $700 \mathrm{nM}$ DHA or E209, as measured by flow cytometry 90 hours later. Data show mean \pm s.e.m. percentage survival compared to DMSO-treated parasites processed in parallel. Assays were performed on at least 2 separate occasions in duplicate. K13-propeller mutations R539T and C580Y confer resistance to DHA in the clinical isolate Cam3.II and the reference line V1/S (a), (b). Exposure to E209 reveals generally higher survival with slight crossresistance observed in parasites carrying the R539T mutation (c), (d). A two-sample t-test with unequal variances was used to calculate $P$ values. These can be found in Supplementary Tables 7-14. ${ }^{\star} P$ value $<0.05,{ }^{\star \star} P$ value $<0.001,{ }^{\star \star \star} P$ value $<0.0001$.

exposure values $\left(C_{\max }\right.$ and $\left.\mathrm{AUC}_{0-24 \mathrm{~h}}\right)$ at the NOAEL on day 7 were $1.2 \mu \mathrm{g} \mathrm{ml}^{-1}$ and $19.6 \mu \mathrm{g} \mathrm{hml}^{-1}$, respectively, in males and $1.07 \mu \mathrm{g} \mathrm{ml}^{-1}$ and $14.8 \mu \mathrm{g} \mathrm{hml}^{-1}$, respectively, in females. Additional single and multiple dose safety pharmacology studies are in progress in rodent and non-rodent models to establish toxicity profile and predicted exposure multiple at NOAEL over target therapeutic exposure (therapeutic index).

\section{Discussion}

A systematic evaluation of the antimalarial tetraoxane scaffold (Supplementary Note 1), revealed the aryloxy template (Fig. 1) to be the most promising in terms of multi-parameter leadoptimization. Through a series of rational chemical modifications we have been able to improve both the inherent pharmacodymamics (in vitro and in vivo potency) and PKs (metabolic 
a

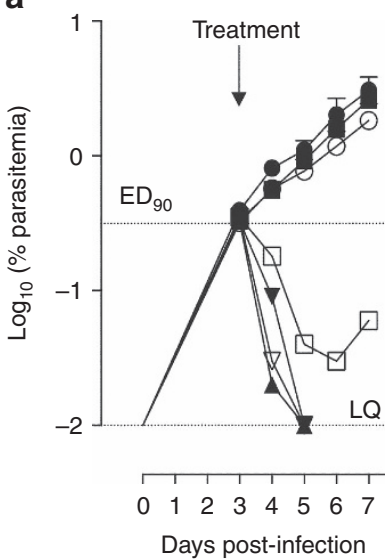

b

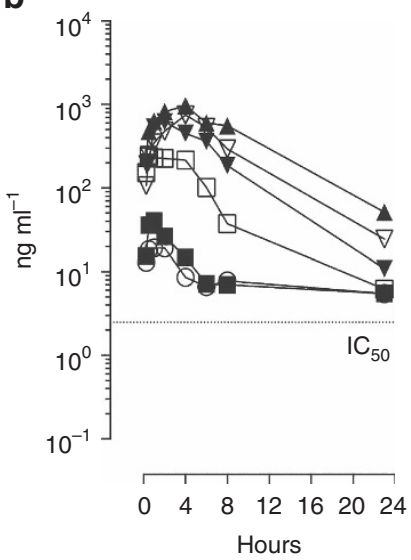

C

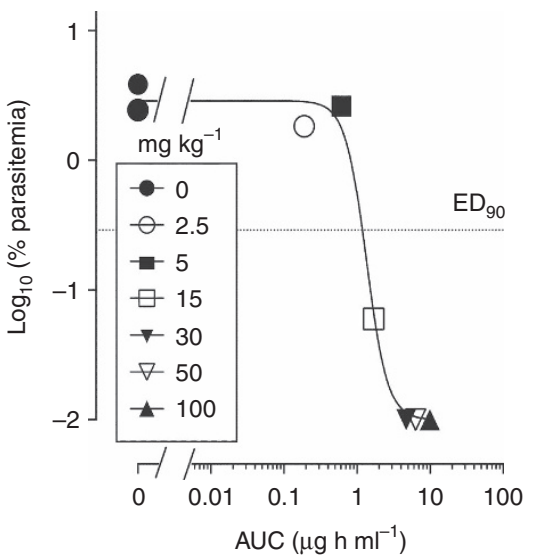

Figure 4 | Efficacy of E209 in the in vivo-humanized SCID mouse model of $\boldsymbol{P}$. falciparum. (a) Parasitemia in peripheral blood of NSG mice infected with the $P$. falciparum strain 3D70087/N9 ( $n=2$ mice treated with vehicle and $n=6$ mice treated with E209). (b) Blood levels of E209 in the efficacy experiment shown in a over the first $24 \mathrm{~h}$ post-dosing. (c) dose/exposure-response relationship.

\begin{tabular}{|c|c|c|c|c|}
\hline & Rat (mean) & Mouse (mean) & Dog ${ }^{\star}$ (mean) & Human (mean) \\
\hline IV dose $\left(\mathrm{mg} \mathrm{kg}^{-1}\right)$ & 2 & 2 & 1 & $\mathrm{~N} / \mathrm{A}$ \\
\hline PO dose $\left(\mathrm{mg} \mathrm{kg}^{-1}\right)$ & 9 & 10 & 5 & $15 \mathrm{mg}^{\dagger}$ \\
\hline Central clearance, $\mathrm{CL}\left(\mathrm{Ih}^{-1} \mathrm{~kg}^{-1}\right)$ & 1.6 & 4.3 & 1.1 & 0.41 \\
\hline Inter-compartmental clearance $2, \mathrm{Q}_{2}\left(\mathrm{Ih}^{-1} \mathrm{~kg}^{-1}\right)$ & 0.08 & 0.97 & 0.13 & 0.020 \\
\hline Peripheral Volume of distribution $1, V_{\mathrm{p} 1}\left(\mathrm{~kg}^{-1}\right)$ & 2.1 & 1.49 & 0.37 & $2.1^{\dagger}$ \\
\hline Peripheral volume of distribution $2, V_{\mathrm{p} 2}\left(\mathrm{~kg}^{-1}\right)$ & 2.0 & 10.8 & 2.95 & $2.0^{\dagger}$ \\
\hline Absorption rate constant $K_{\mathrm{a}}\left(\mathrm{h}^{-1}\right)$ & 0.31 & 0.79 & 0.33 & $0.31^{\dagger}$ \\
\hline$F(\%)$ & 62 & 82 & 40 & $62^{\dagger}$ \\
\hline \multicolumn{5}{|l|}{ In vitro microsomal data } \\
\hline
\end{tabular}

stability profiles in a range of species and rodent and human blood stability) of the class.

In addition to overall high potency, our studies also demonstrate that the fast PRR for E209 observed in in vitro studies translates to a fast action in vivo in the humanized SCID mouse model where a maximum parasite clearance was achieved after a single dose of $30 \mathrm{mg} \mathrm{kg}^{-1}$. To achieve this level of parasite clearance with the clinical gold standard endoperoxide artesunate requires four doses of artesunate $\left(50 \mathrm{mg} \mathrm{kg}^{-1} \mathrm{day}^{-1}\right)$ in the same model ${ }^{25}$. Initial safety and pharmacology profiles for E209 meet the requirements of MMV's target candidate profile 1 (TCP1), a drug with a fast killing profile (PRR equivalent to or better than dihydroartemisinin) and PK qualities that would support a single-dose cure either singly or in combination.

It is generally accepted that the mechanism of action of endoperoxide anti-malarials involves intra-parasitic ferrous mediated reductive scission of the endoperoxide bond. This process leads to the generation of reactive oxygen species such as carbon-centered radicals, which react with multiple parasite proteins and result in parasite killing ${ }^{28-30}$. Because of this proposed mechanism of action it was originally argued that malaria parasites would find it difficult to acquire resistance to this drug class. However, compelling clinical data from Southeast Asia has confirmed that resistance has emerged and established itself in the region. The resistance phenotype has been difficult to study, as it does not show up as a shift in potency $\left(\mathrm{IC}_{50}\right.$ value) in traditional drug sensitivity assays ${ }^{20}$. Extensive molecular investigations have implicated mutations in the K13 gene as key to this unusual resistance phenotype. These mutations allow the parasite to survive exposure to drug at the early ring stage of red blood cell infection even at supra-pharmacological drug concentrations ${ }^{20,21}$. A concern is that this type of resistance mechanism would blight the long-term clinical utility of the entire endoperoxide class of antimalarial drugs. Our interpretation of this resistance mechanism, which is supported by recent modelling studies ${ }^{28}$, is that the slow parasite clearance phenotype seen clinically is a result of the loss of drug susceptibility in ring-stage parasites coupled with the extremely short elimination half-life of the endoperoxide. A potential solution to this problem is to extend the current 3-day artemisinin treatment course to four days as suggested by 
a

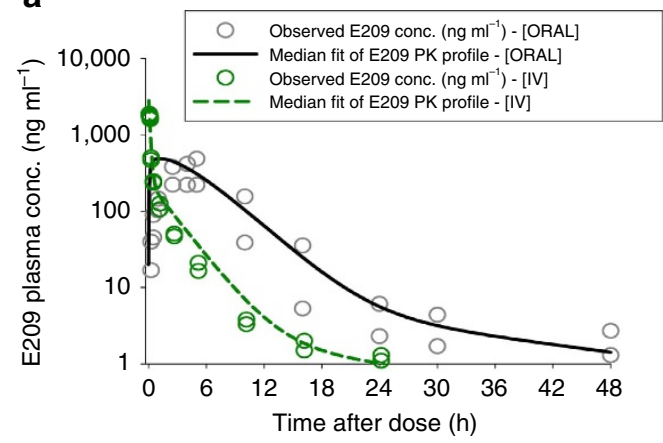

C

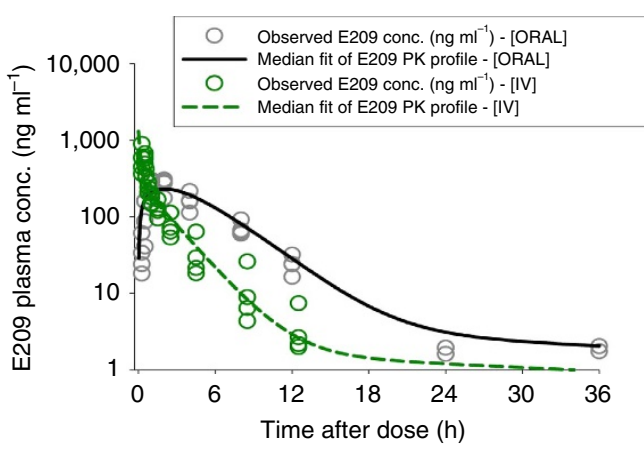

b
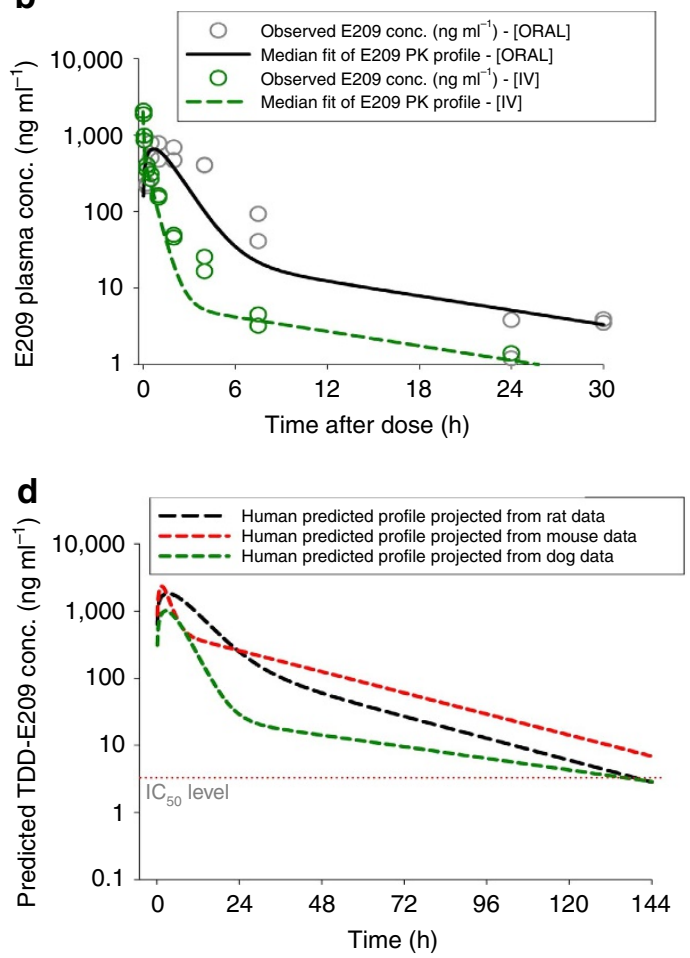

Figure 5 | Measured rodent and predicted human exposure profiles. PK profiles of E209 in male Sprague-Dawley rats (a oral dose $=9 \mathrm{mg} \mathrm{kg}^{-1}, \mathrm{IV}$ dose $\left.=2 \mathrm{mg} \mathrm{kg}^{-1}\right)$, outbred female Swiss mice (b oral dose $=10 \mathrm{mg} \mathrm{kg}^{-1}$, IV dose $=2 \mathrm{mg} \mathrm{kg}^{-1}$ ) and the male beagle dog (c oral dose $=5 \mathrm{mg} \mathrm{kg}^{-1}$, IV dose $=1 \mathrm{mg} \mathrm{kg}^{-1}$ ) and allometrically scaled predictions for human PK (d) based on all of the above assuming an oral dose of $15 \mathrm{mg} \mathrm{kg}{ }^{-1}$. Fits constructed are based on predicted median PK parameter values.

Dogovski et al. ${ }^{31}$ We believe that E209 provides a more elegant solution since it circumvents or minimizes the ring-stage resistance seen with currently deployed endoperoxides with a predicted elimination half-life of $24-30 \mathrm{~h}$ compared to current artemisinins that have half-lives of circa 1-2 h (ref. 32). Hence, not only will E209 plasma levels remain above the $\mathrm{IC}_{50}$ level for 4 days or more in malaria patients as required by MMV's TCP1 criteria (based on PK predictions, Supplementary Methods), but it will also retain killing potential throughout each individual stage of parasite's $48 \mathrm{~h}$ intra-erythrocytic cycle. Thus, E209 has the potential for deployment in a superior combination treatment with a partner drug devoid of existing in vivo resistance liabilities (such as the ATP4 inhibitors ${ }^{33,34}$ or DHODH inhibitors ${ }^{35}$ ). E209 has the potential to offer a substantial improvement on currently deployed artemisininbased drug combinations and provide an urgently required alternative TCP1 drug for malaria treatment and elimination programmes.

\section{Methods}

Synthesis of E209. Details of the synthesis and analytical data on E209 are included in Supplementary Note 2.

Parasite culture and drug sensitivity testing. P. falciparum blood stage cultures were maintained by the method of Trager and Jensen ${ }^{36}$. Drug sensitivity during E209 development and QSAR was determined by the method of Winter et al. ${ }^{37}$ Drug sensitivity of E209 against panel of $P$. falciparum strains was determined based on hypoxanthine incorporation ${ }^{38,39}$. Mammalian cell cytotoxicity assays were carried out as previously described ${ }^{40}$

A zinc-finger nuclease (ZFN)-based gene editing approach was employed to specifically introduce or remove K13 mutations from $P$. falciparum lines. Parasites were electroporated with a plasmid containing ZFNs specifically engineered against K13. The nucleases were expressed from an episomally maintained plasmid by selecting with $2.5 \mathrm{nM}$ (Cam3.II) or $10 \mathrm{nM}$ (V1/S) WR99210 for 6 days. A $1.5 \mathrm{~kb}$ $\mathrm{K} 13$ donor fragment harbouring the desired mutation was placed on the same plasmid and served as template for the double strand break repair. Parasite clones were generated by limiting dilution. A detailed protocol can be found in Straimer et al. ${ }^{21}$

Ring-stage survival assays ( $\mathrm{RSA}_{0-3 \mathrm{~h}}$ ) were carried out as previously described with minor modifications ${ }^{21,23}$. In summary, $10-15 \mathrm{ml}$ parasite cultures were synchronized 1-2 times using 5\% sorbitol (Sigma-Aldrich). Synchronous multinucleated schizonts were incubated in RPMI-1640 containing 15 units $/ \mathrm{ml}$ sodium heparin for $15 \mathrm{~min}$ at $37^{\circ} \mathrm{C}$ to disrupt agglutinated erythrocytes (purchased from Interstate Blood Bank located in Memphis, TN, USA), concentrated over a gradient of 75\% Percoll (Sigma-Aldrich), washed once in RPMI-1640 and incubated for $3 \mathrm{~h}$ with fresh erythrocytes to allow time for merozoite invasion. Cultures were then subjected again to sorbitol treatment to eliminate remaining schizonts. The $0-3 \mathrm{~h}$ post-invasion rings were adjusted to $1 \%$ parasitemia and $2 \%$ haematocrit in $1 \mathrm{ml}$ volumes (in 48 -well plates), and exposed to $700 \mathrm{nM} \mathrm{DHA}$, TDD-E209, JC3-39 or 0.1\% DMSO (solvent control) for $6 \mathrm{~h}$. Duplicate wells were established for each parasite line \pm drug. The supernatant was then removed, the culture resuspended in $1 \mathrm{ml}$ culture media and transferred to $15 \mathrm{ml}$ conical tubes, centrifuged at $800 \mathrm{~g}$ for $3 \mathrm{~min}$ to pellet the cells and the supernatants carefully removed. As a washing step to remove drug, $10 \mathrm{ml}$ culture medium were added to each tube and the cells resuspended, centrifuged and the medium aspirated. This washing procedure was repeated three times and included a transfer into a new $15 \mathrm{ml}$ conical tube after the second washing step. Fresh medium lacking drug was then added to cultures, which were returned to standard culture conditions for $90 \mathrm{~h}$. Parasite viability was assessed by microscopic examination of Giemsa-stained thin blood smears. Second-generation ring stages are clearly visible under the microscope but only give a faint signal when measured by flow cytometry. Therefore, we performed a media change $66 \mathrm{~h}$ after the drug incubation and allowed the culture to develop for another $24 \mathrm{~h}$ into second-generation trophozoite stages. Parasite survival was determined by staining parasites with $2 \times$ SYBR Green I and $165 \mathrm{nM}$ MitoTracker Deep Red (Invitrogen) and a minimum of 8,000 cells were counted by flow cytometry on an Accuri C6 cytometer. Percentage survival was calculated as the parasitemia in the drug-treated sample divided by the parasitemia in the untreated sample $\times 100$.

$P$. cynomolgi $\mathrm{M}$ strain (a non-human primate malaria closely related to the human malaria $P$. vivax and forming dormant liver stages) was used for in vitro liver stage drug assays (The M strain BPRC was obtained from Centers for Disease Control and Prevention (CDC, USA). Liver stage drug assays were performed in primary rhesus monkey hepatocytes as described ${ }^{41}$. Read out of the assay was performed using a high-content imaging system (Operetta) and analysed with Harmony software. As described previously ${ }^{42}$ two populations of liver stage parasite can be discriminated in 6-day-old cultures: large forms define liver 
schizonts and persistent small forms are dormant liver stages (hypnozoites). Small forms are defined as having a maximum parasite area of $30 \mu \mathrm{m}^{2}$.

Ex vivo P. falciparum and P. vivax drug sensitivity experiments were carried out in Papua Indonesia, an area with documented multidrug-resistant $P$. falciparum and CQ-resistant $P$. vivax., using a modified schizont maturation assay as described previously ${ }^{13-17}$. In brief, $200 \mu \mathrm{l}$ of a $2 \%$ haematocrit blood media mixture (BMM), consisting of RPMI- 1640 medium plus $10 \% \mathrm{AB}^{+}$human serum (P. falciparum) or McCoy's $5 \mathrm{~A}$ medium plus $20 \% \mathrm{AB}^{+}$human serum $(P$. vivax $)$ was added to each well of pre-dosed drug plates containing 11 serial concentrations (2-fold dilutions) of the anti-malarials being tested. A candle jar was used to mature the parasites at $37^{\circ} \mathrm{C}$ for $35-56 \mathrm{~h}$. Incubation was stopped when $>40 \%$ of ring-stage parasites had reached mature schizont stage in the drug-free control wells. Thick blood films made from each well were stained with 5\% Giemsa solution for $30 \mathrm{~min}$ and examined microscopically. The number of schizonts per 200 asexual stage parasites was determined for each drug concentration and normalized to the control well. The dose-response data were analysed using nonlinear regression analysis (WinNonLn 4.1, Pharsight) and the $\mathrm{IC}_{50}$ value derived using an inhibitory sigmoid Emax model.

Ethical approval for the ex vivo P. falciparum and $P$. vivax efficacy data was obtained from the Human Research Ethics Committee of the NT Department of Health \& Families and Menzies School of Health Research, Darwin, Australia (HREC 2010-1396) and the Eijkman Institute Research Ethics Commission, Jakarta, Indonesia (EIREC 47 and EIREC 67). Written informed consent was obtained from all patients participating in the study.

The SMFA study was conducted as previously described where mature gametocytes were incubated for $24 \mathrm{~h}$ with different concentrations of E209 prior to performing mosquito feeds ${ }^{43-45}$. The $\mathrm{RSA}_{0-3 \mathrm{~h}}$ was performed as described in Witkowski et al., ${ }^{21}$ whereby tightly synchronized early ring-stage parasites were exposed to a drug pulse for $6 \mathrm{~h}$ at $700 \mathrm{nM}$. After drug removal (by washing) the parasites were returned to culture. Parasitemias were then assessed $66 \mathrm{~h}$ later on thin blood smears by two independent microscopists and the percentage survival was assessed in comparison to parasites not exposed to drug, as described by Straimer et al..$^{23}$ Assays were performed in duplicate on three separate occasions. Parasite reduction ratios were conducted as described in Supplementary Methods ${ }^{20}$

In vivo efficacy. Studies of murine $P$. falciparum infection were ethically reviewed and carried out in accordance with European Directive 2010/63/EU and the GSK Policy on the Care, Welfare and Treatment of Laboratory Animals. In vivo efficacy against $P$. falciparum was conducted ${ }^{25}$ in age-matched female immunodeficient NOD.Cg-Prkdcscid Il2rgtm 1 Wjl/SzJ mice (8-10 weeks of age; $22-24 \mathrm{~g}$ ) supplied by Charles River, UK, under licence of The Jackson Laboratory, Bar Harbor. Mice were engrafted with human erythrocytes (Red Cross Transfusion Blood Bank in Madrid, Spain) by daily intraperitoneal injection with $1 \mathrm{ml}$ of a $50 \%$ haematocrit erythrocyte suspension (RPMI-1640 (Invitrogen), $25 \mathrm{mM}$ HEPES (Sigma), 25\% decomplemented $\mathrm{AB}+$ human serum (Sigma) and $3.1 \mathrm{mM}$ hypoxanthine (Sigma)). Mice with $\sim 40 \%$ circulating human erythrocytes were intravenously infected with $2 \times 10^{7} \mathrm{P}$. falciparum Pf3D $7^{0087 / \mathrm{N} 9}$-infected erythrocytes (day 0). Efficacy was assessed by administering one oral dose of E209 $(2.5,5,15,30,50$ and $100 \mathrm{mg} \mathrm{kg}^{-1}$ ) at day 3 after infection. Treatment group assignments were allocated randomly. Parasitemia was measured by flow cytometry in samples of peripheral blood stained with the fluorescent nucleic acid dye SYTO-16 (Molecular Probes) and anti-murine erythrocyte TER119 monoclonal antibody (Becton Dickinson) in serial $2 \mu \mathrm{l}$ blood samples taken every $24 \mathrm{~h}$ until assay completion. The ED90 was estimated by fitting a four parameter logistic equation using GraphPad 6.0 Software.

Systemic exposure measurement in infected Pf SCID mice. The levels of E209 were evaluated in whole blood to determine standard PK parameters in the individual animals used in the efficacy study. Peripheral blood samples $\left(5 \mu \mathrm{g} \mathrm{ml}^{-1}\right)$ were taken at different times $(0.25,0.5,1,2,4,6,8$ and $23 \mathrm{~h})$ after drug administration, mixed with $25 \mu \mathrm{l}$ of Milli-Q water and immediately frozen on dry ice. The frozen samples were stored at $-80^{\circ} \mathrm{C}$ until analysis. Vehicle-treated mice experienced the same blood-sampling regimen. Blood samples were processed by liquid-liquid extraction. Quantitative analysis by Liquid chromatography-tandem mass spectrometry (LC-MS/MS) was performed using a Waters UPLC system and Sciex API4000 mass spectrometer. The lower limit of quantification in this assay was $5 \mathrm{ng} \mathrm{ml}^{-1}$. Blood concentration versus time was analysed by noncompartmental analysis (NCA) using Phoenix ver.6.3 (from Pharsight), from which exposure-related values $\left(C_{\max }\right.$ and $\left.\mathrm{AUC}_{0-23}, \mathrm{AUC}_{0-t}\right)$ and $t_{\max }$ were estimated.

In vitro and in vivo PK studies. Details of the in vitro PK analysis of E209 are contained in Supplementary Tables 1, 2, 3, 17, 19 and 19). In vitro metabolic stability of E209 was carried out as described in Supplementary Methods.

In vivo $\mathrm{PK}$ studies in rats and mice were conducted using established procedures in accordance with the Australian Code of Practice for the Care and Use of Animals for Scientific Purposes, and the study protocols were reviewed and approved by the Monash Institute of Pharmaceutical Sciences Animal Ethics Committee.

Rat PK studies were conducted in fasted 7-9-week-old male Sprague-Dawley rats, as previously described by Charman et al. ${ }^{12}$ For intravenous administration E209 was dissolved in 5\% glucose solution under sonication (for $10 \mathrm{~min}$ ) prior to addition of Tween 80 and further sonication $(5 \mathrm{~min})$ to produce a clear solution. The formulation was filtered through a $0.22 \mu \mathrm{m}$ syringe filter prior to dosing and aliquots retained to determine the dose administered. E209 was administered intravenously as a $10 \mathrm{~min}$ constant rate infusion via an indwelling jugular vein cannula ( $1 \mathrm{ml}$ per rat, $n=2$ rats).

For oral dosing, E209 was dissolved in $0.5 \%$ [w/v] hydroxypropyl methylcellulose containing $0.4 \%[\mathrm{w} / \mathrm{v}]$ Tween 80 and $0.5 \%[\mathrm{v} / \mathrm{v}]$ benzyl alcohol and sonicated for $5 \mathrm{~min}$ producing a clear solution. The bulk formulation was mixed by inverting the tubes prior to drawing each dosing volume. Aliquots of the dosing solution were retained for analysis to determine the dose administered. After the oral formulation ( $1 \mathrm{ml}$ per rat, $n=2$ rats) was administered, an additional volume (1 ml) of Milli-Q water was administered (via a separate syringe).

Blood samples were collected using a Culex automated blood sampler over $48 \mathrm{~h}$ post-dosing into tubes containing anticoagulant and stabilization cocktail (Complete, potassium fluoride and EDTA) to minimize the potential for ex vivo degradation and immediately centrifuged at $4{ }^{\circ} \mathrm{C}$. Plasma was separated, stored at $-20^{\circ} \mathrm{C}$ until processing and assayed by LC/MS using either a Waters Micromass Quattro Ultima PT triple quadrupole instrument coupled to a Waters 2795 HPLC or a Waters Micromass Quattro Premier triple quadrupole instrument coupled to a Waters Acquity UPLC

The PKs of E209 was also studied in non-fasted 6-8 week old female Swiss Outbred mice that had access to food and water ad libitum throughout the pre- and post-dose sampling period. The formulations for intravenous and oral administration were the same as used for the rat studies (study protocols were reviewed and approved by the Monash Institute of Pharmaceutical Sciences Animal Ethics Committee).

E209 was administered intravenously by bolus tail vein injection ( $50 \mu \mathrm{l}$ per mouse) or orally by gavage $\left(10 \mathrm{ml} \mathrm{kg}^{-1}\right)$ and blood samples collected over $30 \mathrm{~h}$ post-dosing ( $n=2$ mice per time point). A maximum of two samples were obtained from each mouse, with samples being taken either via submandibular bleed (conscious sampling) or terminal cardiac puncture (underinhaled isofluorane anaesthesia). Blood was collected into tubes in the same way as described for the rat studies and immediately centrifuged and stored at $-20^{\circ} \mathrm{C}$ until analysis by LC-MS.

Plasma PK parameters were calculated based on compartmental PK analysis using Pmetrics ${ }^{46}$ as described in Supplementary Methods. A classical three compartment oral absorption model was used for all tested species due to the tri-phasic profiles displayed over time. For human predictions, allometric scaling was performed based on weight. Clearance rates (including inter-compartmental clearance rates Q1 and Q2) were predicted according to the following equations:

$$
\begin{aligned}
\mathrm{CL}_{\text {human }} & =\mathrm{CL}_{\text {animal }} \times\left[\frac{\text { Humanweight }}{\text { Animalweight }}\right]^{0.75} \\
\mathrm{Q}_{\text {human }} & =\mathrm{Q}_{\text {animal }} \times\left[\frac{\text { Humanweight }}{\text { Animalweight }}\right]^{0.75}
\end{aligned}
$$

Other PK parameters were scaled linearly and simulation of human exposure to TDD-E209 was performed assuming a $15 \mathrm{mg} \mathrm{kg}^{-1}$ dose using Pmetrics where median values were plotted over time on a semi-log scale.

hERG profiling and assessment of cytotoxicity. The potencies ( $\mathrm{IC}_{50}$ values) for E209 to inhibit the cardiac $\mathrm{I}_{\mathrm{Kr}}$ (hERG), $\mathrm{Cav}_{1.2}$ and $\mathrm{Nav}_{1.5}$ channels were determined in an electrophysiology-based assays using IonWorks HT (CHO cells $)^{27}$

In vivo (rat) safety studies. A single, rising oral dose study to determine the MTD of E209 and aid in the selection of dose levels for the 7-day toxicity study. The rats (Sprague-Dawley, age 6-7 weeks) were divided into four groups, each with three male and three female. Dose levels employed were 30, 100, 300 and $1,000 \mathrm{mg} \mathrm{kg}^{-1}$ and the MTD was shown to be $300 \mathrm{mg} \mathrm{kg}^{-1}$.

Following this single dose MTD study, rats were dosed once a day at 0,30,100 and $300 \mathrm{mg} \mathrm{kg}^{-1}$ po E209 for 7 days. E209 was formulated in $0.5 \%$ [w/v] HPMC, $0.4 \%$ Tween $80[\mathrm{w} / \mathrm{v}], 0.5 \%[\mathrm{v} / \mathrm{v}]$ benzyl alcohol in purified water. In each group there were eight Sprague-Dawley rats of each sex. At the end of the dosing period, five rats/sex/group were killed (day 8) and the remaining three rats/sex/group were left for recovery. In addition, six rats of each sex per group were allocated for toxicokinetics.

In vivo PK studies conformed to AAALAC International and NIH guidelines as reported in the Guide for the Care and Use of Laboratory Animals, National Research Council (2011); People's Republic of China, Ministry of Science \& Technology, 'Regulations for the Administration of Affairs Concerning Experimental Animals', 1988.

Data availability. All relevant data are available from the authors upon request. 


\section{References}

1. WHO. Global Report on Antimalarial Drug Efficacy and Drug Resistance: 2000-2010 (WHO, 2010).

2. WHO. Guidelines for the Treatment of Malaria 2nd edn (WHO, 2010).

3. Wells, T. N., van Huijsduijnen, R. H. \& Van Voorhis, W. C. Malaria medicines: a glass half full? Nat. Rev. Drug. Discov. 14, 424-442 (2015).

4. Noedl, H. et al. Evidence of artemisinin-resistant malaria in western Cambodia. N. Engl. J. Med. 359, 2619-2620 (2008).

5. Dondorp, A. M. et al. Artemisinin resistance in Plasmodium falciparum malaria. N. Engl. J. Med. 361, 455-467 (2009).

6. Bruce-Chwatt, L. J. et al. Chemotherapy of Malaria 2nd edn (World Health Organization, 1986)

7. WHO. Chemotherapy of Malaria. Report of a WHO Scientific Group. WHO Technical Report Series, No. 375 (World Health Organization, 1967).

8. WHO. Status Report on Artemisinin Resistance. Available at: (http:// www.who.int/malaria/publications/atoz/status-rep-artemisinin-resistancesep2014.pdf)(2014).

9. Leang, R. et al. Evidence of Plasmodium falciparum malaria multidrug resistance to artemisinin and piperaquine in western Cambodia: dihydroartemisinin-piperaquine open-label multicenter clinical assessment. Antimicrob. Agents Chemother. 59, 4719-4726 (2015).

10. Gomes, G. d. P., Vil, V., Terent'ev, A. \& Alabugin, I. V. Stereoelectronic source of the anomalous stability of bis-peroxides. Chem. Sci. 6, 6783-6791 (2015).

11. O'Neill, P. M. et al. Identification of a 1,2,4,5-tetraoxane antimalarial drugdevelopment candidate (RKA 182) with superior properties to the semisynthetic artemisinins. Angew. Chem. Int. Ed. Engl. 49, 5693-5697 (2010).

12. Charman, S. A. et al. Synthetic ozonide drug candidate OZ439 offers new hope for a single-dose cure of uncomplicated malaria. Proc. Natl Acad. Sci. USA 108, 4400-4405 (2011).

13. Karyana, M. et al. Malaria morbidity in Papua Indonesia, an area with multidrug resistant Plasmodium vivax and Plasmodium falciparum. Malar. J. 7, 148 (2008).

14. Ratcliff, A. et al. Therapeutic response of multidrug-resistant Plasmodium falciparum and $P$. vivax to chloroquine and sulfadoxine-pyrimethamine in southern Papua, Indonesia. Trans. R. Soc. Trop. Med. Hyg. 101, 351-359 (2007).

15. Russell, B. et al. Determinants of in vitro drug susceptibility testing of Plasmodium vivax. Antimicrob. Agents Chemother. 52, 1040-1045 (2008).

16. Marfurt, J. et al. Ex vivo activity of histone deacetylase inhibitors against multidrug-resistant clinical isolates of Plasmodium falciparum and P. vivax. Antimicrob. Agents Chemother. 55, 961-966 (2011).

17. Marfurt, J. et al. Ex vivo drug susceptibility of ferroquine against chloroquineresistant isolates of Plasmodium falciparum and P. vivax. Antimicrob. Agents Chemother. 55, 4461-4464 (2011).

18. Vos, M. W. et al. A semi-automated luminescence based standard membrane feeding assay identifies novel small molecules that inhibit transmission of malaria parasites by mosquitoes. Sci. Rep. 5, 18704 (2015).

19. Bolscher, J. M. et al. A combination of new screening assays for prioritization of transmission-blocking antimalarials reveals distinct dynamics of marketed and experimental drugs. J. Antimicrob. Chemother. 70, 1357-1366 (2015).

20. Sanz, L. M. et al. P. falciparum in vitro killing rates allow to discriminate between different antimalarial mode-of-action. PLoS ONE 7, e30949 (2012).

21. Witkowski, B. et al. Novel phenotypic assays for the detection of artemisininresistant Plasmodium falciparum malaria in Cambodia: in-vitro and ex-vivo drug-response studies. Lancet Infect. Dis. 13, 1043-1049 (2013).

22. Witkowski, B. et al. Reduced artemisinin susceptibility of Plasmodium falciparum ring stages in western Cambodia. Antimicrob. Agents Chemother. 57, 914-923 (2013).

23. Straimer, J. et al. Drug resistance. K13-propeller mutations confer artemisinin resistance in Plasmodium falciparum clinical isolates. Science 347, 428-431 (2015).

24. Peters, W. \& Robinson, B. L. Malaria (Academic Press, 1999).

25. Angulo-Barturen, I. et al. A murine model of falciparum-malaria by in vivo selection of competent strains in non-myelodepleted mice engrafted with human erythrocytes. PLoS ONE 3, e2252 (2008).

26. Jimenez-Diaz, M. B. et al. Improved murine model of malaria using Plasmodium falciparum competent strains and non-myelodepleted NOD-scid IL2R gamma(null) mice engrafted with human erythrocytes. Antimicrob. Agents Chemother. 53, 4533-4536 (2009).

27. Rochford, R. et al. A humanized (hu)RBC-SCID mouse model of glucose 6-phosphate dehydrogenase deficiency for in vivo assessment of toxic hemolytic responses. Proc. Natl Acad. Sci. USA 110, 17486-17491 (2013).

28. Wang, J. et al. Haem-activated promiscuous targeting of artemisinin in Plasmodium falciparum. Nat. Commun. 6, 10111 (2015).

29. Ismail, H. M. et al. Probing the molecular targets of artemisinins in the malaria parasite: a chemical proteomic approach. Proc. Natl Acad Sci. USA 113, 2080-2085 (2016)
30. Ismail, H. M. et al. A click chemistry-based proteomic approach reveals that 1,2,4-trioxolane and artemisinin antimalarials share a common protein alkylation profile. Angew. Chem. Int. Ed. Engl. 55, 6401-6405 (2016).

31. Dogovski, C. et al. Targeting the cell stress response of Plasmodium falciparum to overcome artemisinin resistance. PLoS Biol. 13, el002132 (2015).

32. Morris, C. A. et al. Review of the clinical pharmacokinetics of artesunate and its active metabolite dihydroartemisinin following intravenous, intramuscular, oral or rectal administration. Malar. J. 10, 263 (2011).

33. Jiménez-Díaz, M. B. et al. (+ )-SJ733, a clinical candidate for malaria that acts through ATP4 to induce rapid host-mediated clearance of Plasmodium. Proc. Natl Acad Sci. USA 111, E5455-E5462 (2014).

34. Rottmann, M. et al. Spiroindolones, a new and potent chemotype for the treatment of malaria. Science 329, 1175-1180 (2010).

35. Coteron, J. M. et al. Structure-guided lead optimization of triazolopyrimidinering substituents identifies potent Plasmodium falciparum dihydroorotate dehydrogenase inhibitors with clinical candidate potential. J. Med. Chem. 54, 5540-5561 (2011).

36. Trager, W. \& Jensen, J. B. Human malaria parasites in continuous culture. Science 193, 673-675 (1976).

37. Winter, R. W. et al. Evaluation and lead optimization of anti-malarial acridones. Exp. Parasitol. 114, 47-56 (2006).

38. Desjardins, R. E. et al. Quantitative assessment of antimalarial activity In vitro by a semiautomated microdilution technique. Antimicrob. Agents Chemother. 16, 710-718 (1979).

39. Snyder, C. et al. In vitro and in vivo interaction of synthetic peroxide RBx11160 (OZ277) with piperaquine in Plasmodium models. Exp. Parasitol. 115, 296-300 (2007).

40. Copple, I. M. et al. Examination of the cytotoxic and embryotoxic potential and underlying mechanisms of next-generation synthetic trioxolane and tetraoxane antimalarials. Mol. Med. 18, 1045-1055 (2012).

41. Zeeman, A.-M. et al. KAI407, a potent non-8-aminoquinoline compound that kills Plasmodium cynomolgi early dormant liver stage parasites in vitro. Antimicrob. Agents Chemother. 58, 1586-1595 (2014).

42. Dembele, L. et al. Persistence and activation of malaria hypnozoites in longterm primary hepatocyte cultures. A potential role for the epigenetic control of quiescence. Nat. Med. 20, 307-312 (2014).

43. McNamara, C. W. et al. Targeting Plasmodium PI(4)K to eliminate malaria. Nature 504, 248-253 (2013).

44. Baragana, B. et al. A novel multiple-stage antimalarial agent that inhibits protein synthesis. Nature 522, 315-320 (2015).

45. Almela, M. J. et al. A new set of chemical starting points with Plasmodium falciparum transmission-blocking potential for antimalarial drug discovery. PloS ONE 10, e0135139 (2015).

46. Neely, M. N., van Guilder, M. G., Yamada, W. M., Schumitzky, A. \& Jelliffe, R. W. Accurate detection of outliers and subpopulations with Pmetrics, a nonparametric and parametric pharmacometric modeling and simulation package for $\mathrm{R}$. Ther. Drug. Monit. 34, 467-476 (2012).

\section{Acknowledgements}

This work was supported by grants from the European Union (Antimal, FP6, S.A.W., P.M.O'N.), the Medicines for Malaria Venture (S.A.W., P.M.O'N., G.A.B., G.L.N., R.K.A.), the NIH (R01 AI109023 to D.A.F.), the Global Health and Innovative Technology (GHIT) Fund (G2015-120, S.A.W., P.M.O'N., G.A.B, G.L.N.) and Medical Research Council, United Kingdom (G0700654, M.H.-L.W., A.E.M., I.M.C.). We would like to thank Dr Sergio Wittlin (Swiss Tropical and Public Health Institute Socinstrasse 57, 4051 Basel, Switzerland) for the data recorded in Table 1 (Plasmodium berghei mouse model).

\section{Author contributions}

P.M.O’N., S.A.W., G.A.B., G.L.N., D.A.F., S.F.B., I.A.-B., G.A., S.A.C., B.C. wrote the manuscript. P.M.O’N., G.L.N., R.K.A., S.S., E.R.S., N.L.R., M.H.-L.W., C.R., C.P., M.J.P., B.M., W.D.H., M.S., S.C. completed the chemistry, design of compounds, synthetic routes and analysis. J.D. carried out the In vitro antimalarial efficacy experiments. G.W., R.N., R.P., J.M. performed the clinical field isolate work; E.R., D.S., G.A., R.S., D.A.S. completed PK/PD modelling and stability studies. F.J.G., L.S. completed the in vitro PRR experiments. J.F.R., C.G. and E.H. completed the Standard Membrane feeding Assay SMFA work. F.J.G., L.S. completed the PRR assays. I.A.-B., M.B.J.-D. completed the in vivo efficacy work in SCID mice. S.F.B., M.S.M. undertook the PK experiments in SCID mice D.F. designed the RSA0-3 $\mathrm{h}$ assays, which were performed by N.G. and J.S., R.S., M.D. and A.R. performed the transmission-blocking studies and TRA analysis. S.A.C., E.R., D.M.S. performed in vitro and in vivo PK studies. R.R. designed and performed G6PD deficient mice experiment. B.C. managed the CRO work, project management and toxicology studies. I.M.C., A.E.M., P.S. carried out in vitro and in vivo toxicology studies. C.K., A.-M.Z. performed liver stage drug assays in primary rhesus monkey hepatocytes 


\section{Additional information}

Supplementary Information accompanies this paper at http://www.nature.com/ naturecommunications

Competing interests: The authors declare no competing financial interests.

Reprints and permission information is available online at http://npg.nature.com/ reprintsandpermissions/

How to cite this article: O'Neill, P. M. et al. A tetraoxane-based antimalarial drug candidate that overcomes PfK13-C580Y dependent artemisinin resistance. Nat.

Commun. 8, 15159 doi: 10.1038/ncomms15159 (2017).
Publisher's note: Springer Nature remains neutral with regard to jurisdictional claims in published maps and institutional affiliations.

(c) (i) This work is licensed under a Creative Commons Attribution 4.0 International License. The images or other third party material in this article are included in the article's Creative Commons license, unless indicated otherwise in the credit line; if the material is not included under the Creative Commons license, users will need to obtain permission from the license holder to reproduce the material. To view a copy of this license, visit http://creativecommons.org/licenses/by/4.0/

C) The Author(s) 2017 